P. Valabrega

Nagoya Math. J.

Vol. 61 (1976), 127-133

\title{
A FEW THEOREMS ON COMPLETION OF EXCELLENT RINGS ${ }^{(1)}$
}

\author{
PAOLO VALABREGA
}

\section{Introduction.}

In [2], chap. IV, $2^{\text {me }}$ partie, (7.4.8), Grothendieck considered the following problem: is any m-adic completion of an excellent ring $A$ also excellent?

In [8] I proved that, if $A$ is an algebra of finite type over an arbitrary field $k$, the answer is positive.

When $A$ is finitely generated over an excellent Dedekind domain $C$ there is a partial positive answer in [5] for $C=$ Dedekind domain of characteristic 0 and with perfect residue fields.

In the present paper I can remove the condition on the residue fields and show that, if $A$ is an algebra of finite type over an excellent ring $C$ of characteristic 0 and dimension 1 , then $(A, \mathfrak{m})^{\wedge}$ is excellent for every ideal $\mathfrak{m}$ of $A$.

The result follows from a proper use of jacobian criteria of regularity and from a theorem I can prove here on the closedness of singular loci. Precisely I can show that, if $R$ is $m$-adically separated and complete, $R / \mathfrak{m}$ is excellent and the singular locus of every finite $R$ algebra is closed, then the formal fibers of $R$ are geometrically regular. Such result allows us also to prove excellent property for some new class of completions of excellent rings ; for instance, if $A$ is a two-dimensional Nagata ring, all m-adic completions of $A$ are excellent rings.

I wish to thank prof. H. Matsumura for many useful conversations on the subject of this paper.

n. 1

Terminology will be the same as in [8] and [3]. Unique exception:

Received October 17, 1975.

(1) The present paper was written while the author was supported by the CNR (section GNSAGA). 
a ring will be called "quasi excellent" when it has all properties of excellent rings except that perhaps it is not universally catenary.

In the present section there are some auxiliary results on Dedekind domains and maximal ideals in polynomial rings.

Proposition 1: Let $C$ be a Nagata Dedekind domain, $\mathfrak{p} \subset C$ a maximal ideal, $K=C / \mathfrak{p}, \tilde{K}$ a finite extension of $K$. Then there are a domain $\tilde{C}$ containing $C$ and a maximal ideal $\tilde{\mathfrak{p}}$ of $\tilde{C}$ such that:

(i) $\tilde{C}$ is a finite $C$-module;

(ii) $\tilde{C}$ is a Nagata Dedekind domain;

(iii) $\tilde{\mathfrak{p}}$ lies over $\mathfrak{p}$;

(iv) $\tilde{C} / \tilde{p}=\tilde{K}$.

Proof: We have: $\tilde{K}=K\left[a_{1}, \cdots, a_{n}\right]$, where the $a_{i}$ 's are algebraic over $K$. Using induction on $n$ we can assume that $n=1$, say $\tilde{K}=K[a]$, with $f(a)=0$ minimal monic equation of $a$ over $K$. Lift $f(T)$ to a monic polynomial $F(T) \in C[T]$ and consider the finite free module $C^{\prime}=$ $C[T] /(F(T))$. Since $C^{\prime} / \mathfrak{p} C^{\prime}=K[T] /(f(T))=K[a]=\tilde{K}, \mathfrak{p} C^{\prime}$ is maximal in $C^{\prime}$. Moreover $C_{p C^{\prime}}^{\prime}$ is a $D V R$ with parameter any parameter of $C_{p}$. Take now $\tilde{C}=$ derived normal ring of $C^{\prime}, \tilde{\mathfrak{p}}=$ minimal prime over $\mathfrak{p} C^{\prime}$, hence over $\mathfrak{p}$. Then $\tilde{C}_{\tilde{p}}=C_{\mathfrak{p} C^{\prime}}^{\prime}$, since $C^{\prime}$ is normal at $\mathfrak{p} C^{\prime}$ ([7], chap. V, (33.1)); so $\tilde{C} / \tilde{\mathfrak{p}}=C^{\prime} / \mathfrak{p} C^{\prime}=\tilde{K}$.

$\tilde{C}$ is finite over $C$, since $C$ is Nagata. So $\tilde{C}$ is Nagata.

Remark: Of course, if $C$ is excellent, then also $\tilde{C}$ is excellent.

Propositiom 2: Let $C$ be a Dedekind domain, $A=C\left[X_{1}, \cdots, X_{n}\right]=$ $C[\underline{X}]$ a polynomial ring and $\mathfrak{M}$ a maximal ideal of $A$ such that $\mathfrak{M} \cap C=$ (0). Then $\mathfrak{M}$ has height $n$.

Proof: Induction on $n$. First of all, if $n=1$, any maximal ideal of $A$ lying over (0) must have height 1, by [9], appendix 1, proposition 1. In dimension $n$ we know that $\mathfrak{M}^{\prime}=\mathfrak{M} \cap C\left[X_{1}, \cdots, X_{n-1}\right]$ is maximal and so we have: $\operatorname{ht}\left(\mathfrak{M}^{\prime}\right)=n-1$. Moreover $\mathfrak{M} \neq \mathfrak{M}^{\prime} A$, so that $\operatorname{ht}(\mathfrak{M})=$ ht( $\left.\mathfrak{M}^{\prime}\right)+1$ ([9], loc. cit.).

Proposition 3: Let $K$ be a field, $A=K\left[X_{1}, \cdots, X_{n}\right]=K[\underline{X}]$ a polynomial ring and $\mathfrak{M}$ a maximal ideal of $A$. Then there are a field $\tilde{K}$, finite extension of $K$, and a maximal ideal $\tilde{\mathfrak{M}}$ of $\tilde{K}[\underline{X}]$ such that:

(i) $\mathfrak{M} \tilde{K}[\underline{X}] \subseteq \tilde{\mathfrak{M}}$; 
(ii) $\tilde{\mathfrak{M}}=\left(X_{1}-a_{1}, \cdots, X_{n}-a_{n}\right) \tilde{K}[\underline{X}]$, where the $a_{i}$ 's are suitable elements in $\tilde{K}$.

Proof: By Hilbert's Nullstellensatz $\mathfrak{M}$ has an algebraic zero $\left(a_{1}\right.$, $\left.\cdots, a_{n}\right)$ in $\bar{K}^{n}$, where $\bar{K}$ is an algebraic closure of $K$. But such a zero belongs to the finite extension $\tilde{K}=K[\underline{a}]$. Then $\tilde{\mathfrak{M}}=\left(X_{1}-a_{1}, \cdots, X_{n}-\right.$ $\left.a_{n}\right) \tilde{K}[\underline{X}]$ is the required ideal.

n. 2

In the present section we prove some results on the closedness of singular loci for $\mathfrak{\Im}$-adically complete rings.

THEOREM 4: Let $R$ be a noetherian ring and $\mathfrak{\Im}$ an ideal such that:

(i) $R$ is $\mathfrak{\Im}$-adically separated and complete;

(ii) $R / \mathfrak{S}$ is quasi excellent;

(iii) $R$ is $J-2$.

Then $R$ is quasi excellent.

Proof: Let $\mathfrak{P}$ be a prime ideal of $R$ : we have to show that $R / \mathfrak{P}$ is a G-ring. If $\mathfrak{J} \subseteq \mathfrak{B}$ there is nothing to prove. Therefore we can assume that $\mathfrak{S}$ is not contained in $\mathfrak{P}$, so that $R / \mathfrak{P}$ is again $\mathfrak{J}$-adically separated and complete. Hence we can assume that $R$ is a domain satisfying conditions (i)-(iii).

Let $\mathfrak{M}$ be a maximal ideal of $R$ and put $C=R_{\mathfrak{M}}$. By [3], chap. XIII (33. E), lemma 3, it is enough to show that, if $D$ is a domain finite as a $C$-module and $\mathfrak{Q}$ is a prime ideal of $\hat{D}=(D, \operatorname{Rad}(D))^{\wedge}$ such that $\mathfrak{Q} \cap D=(0)$, then the local ring $\hat{D}_{\mathfrak{a}}$ is regular.

Put: $X=\operatorname{Spec}(D), X^{\prime}=\operatorname{Spec}(\hat{D})$ and let $f: X^{\prime} \rightarrow X$ be the canonical map. Then it is enough to show that $f^{-1}(\operatorname{Reg}(X)) \subseteq \operatorname{Reg}\left(X^{\prime}\right)$.

Assume that $Y=f^{-1}(\operatorname{Reg}(X)) \cap$ Sing $\left(X^{\prime}\right) \neq \emptyset$. Since $R$ is $J-2, D$ is $J-1$, i.e. the set $\operatorname{Reg}(X)$ is open in $X$. On the other hand Sing $\left(X^{\prime}\right)$ is closed, since $\hat{D}$ is a complete semilocal ring (Nagata's jacobian criteria). Therefore $Y$ is locally closed and not empty, which implies that it contains a prime ideal $\mathfrak{p}^{\prime}$ such that $\operatorname{dim}\left(\hat{D} / \mathfrak{p}^{\prime}\right) \leq 1$ ([3], chap. XIII, (33. F), lemma 5). Since $\mathfrak{p}^{\prime}$ cannot be maximal, we have: $\operatorname{dim}\left(\hat{D} / \mathfrak{p}^{\prime}\right)=1$. Now $\hat{D}_{\mathfrak{p}^{\prime}}$ is singular, while $D_{\mathfrak{p}}$ is regular, if $\mathfrak{p}=\mathfrak{p}^{\prime} \cap D$. So also the local ring $\hat{D}_{\mathfrak{p}^{\prime}} / \mathfrak{p} \hat{D}_{\mathfrak{p}^{\prime}}$ is singular by [3], (21. D), theorem 51). From these facts we want to deduce a contradiction.

Put: $E=\hat{D} / \mathfrak{p}^{\prime}, \mathfrak{S}^{\prime}=R \cap \mathfrak{p}^{\prime}=R \cap \mathfrak{p}$, so that $R / \mathfrak{S}^{\prime} \longrightarrow D / \mathfrak{p} \hookrightarrow E$ are 
inclusions of domains. Moreover put: $\mathfrak{m}_{A}=$ Radical of $A$, for any ring $A$ we have to consider.

Considering the ideal $\mathfrak{\Im} E$ we have two alternatives:

(i) $\widetilde{\Im} E=(0)$ : this means that $\mathfrak{\Im} \hat{D} \subseteq \mathfrak{p}^{\prime}$ hence $\mathfrak{\Im} D \subseteq \mathfrak{p}$, so that $D / \mathfrak{p}$ is a finite $R / \mathfrak{S}$-module. But $R / \mathfrak{J}$ is quasi excellent, hence $D / \mathfrak{p}$ is quasi excellent, which means in particular that $\hat{D}_{\mathfrak{p}^{\prime}} / \mathfrak{p} \hat{D}_{\mathfrak{p}^{\prime}}$ must be a regular local ring; but this is absurd.

(ii) $\mathfrak{S} E \neq(0)$ : since $E$ is a noetherian local domain of dimension 1 , $\Im E$ defines its natural topology. In other words there is an integer $r>0$ such that $\mathfrak{m}_{E}^{r} \subseteq \mathfrak{S} E$. Now $E / \mathfrak{S} E$ is a homomorphic image of $E / \mathfrak{m}_{E}^{r}=$ homomorphic image of $\hat{D} / \mathfrak{m}_{\widehat{D}}^{r}=D / \mathfrak{m}_{D}^{r}=$ finite module over $C / \mathfrak{m}_{C}^{r}=R / \mathfrak{M}^{r}$. Finally $E / \mathfrak{S} E$ is a finite module over $R$. Since $R$ is $\mathfrak{\Im}$-adically separated and complete $E$ is finite over $R$ or, which is the same, over $R / \mathfrak{J}^{\prime}$. Hence $R / \mathfrak{S}^{\prime} \longrightarrow D / \mathfrak{p} \longrightarrow E$ are all finite inclusions of one-dimensional domains. But $D / p$ is a Nagata ring by [6], proposition 1 ; hence, being one-dimensional, it is quasi excellent. But this fact is absurd.

Finally $Y=\emptyset$, which means that $R$ is a $G$-ring.

Here now a list of the main corollaries:

COROLLARY 5: Let $R$ be a noetherian domain and $\mathfrak{S}$ a non zero ideal of $R$ such that: (i) $\operatorname{dim}(R)=2$; (ii) $R$ is $\mathfrak{S}$-adically separated and complete; (iii) $R / \mathfrak{S}$ is a Nagata ring. Then $R$ is quasi excellent.

Proof: Since $\operatorname{dim}(R / \mathfrak{S}) \leq 1, R / \mathfrak{S}$ is quasi excellent. Consider now a domain $D$ which is a finite $R$-module. If $\operatorname{dim}(D) \leq 1$, then $D$ is quasi excellent, hence $J-1$, because it is Nagata ([6], proposition 1). If $\operatorname{dim}(D)=2$, there is a non zero element $f \in \operatorname{Rad}(D)$; hence $D_{f}$ is a onedimensional Nagata domain ([6], proposition 1), so that $D_{f}$ is $J-1$ $\left(\operatorname{Reg}\left(D_{f}\right)=\operatorname{Nor}\left(D_{f}\right)=\right.$ open set).

Therefore $R$ is $J-2$ and also quasi excellent by theorem 4 .

COROLlaRY 6: Let $R$ be a Nagata domain of dimension 2. Then any proper $\mathfrak{\Im}$-adic completion of $R$ is quasi excellent.

Proof: immediate from corollary 5.

COROLlaRY 7: Let $R$ be a regular domain of dimension 3 and characteristic 0 . If $R$ is $\mathfrak{\Im}$-adically separated and complete for some ideal $\mathfrak{\Im}$ and $R / \mathfrak{S}$ is excellent, then $R$ is excellent. 
Proof: No problem for universal catenarianness, since $R$ is regular. If $\mathfrak{P}$ is a prime ideal such that $\operatorname{dim}(R / \mathfrak{P}) \leq 2$, then either $\mathfrak{J} \subseteq \mathfrak{P}$ and we are done by hypothesis, or $\mathfrak{S} \nsubseteq \mathfrak{P}$, so that we can apply corollary 5 . Moreover there is no problem at the origin since $R$ is supposed regular.

COROLLARY 8: Let $R$ be a quasi excellent ring of dimension 3 such that $R / \Re$ has characteristic 0 for every prime $\mathfrak{\beta}$ of height 0 . Then any $\widetilde{\mathcal{S}}$-adic completion of $R$ is quasi excellent.

Proof: $\operatorname{Reg}(\hat{R} / \mathfrak{\Re})$ is open for every prime of height 0 in $\hat{R}$, since $R \hookrightarrow \hat{R}$ is a regular map. As far as prime ideals of height greater than 0 are concerned, we can apply corollary 5 .

Remark: In corollary 7 and 8 we consider rings containing the ring $Z$ of integers, but not necessarily the field of rational numbers.

n. 3

In the present section we prove our main theorem on the excellent property for the m-adic completions of algebras of finite type over excellent Dedekind domains, obtaining a generalization of the main result given in [5], where the residue fields of the base ring are supposed to be perfect.

THeorem 9: Let $C$ be an excellent ring of dimension 1 and such that $C / \Re$ has characteristic 0 (but perhaps unequal characteristic) for every $\mathfrak{P}$ of height 0 . Then any $\mathfrak{m}$-adic completion of an algebra of finite type over $C$ is an excellent ring.

Proof: Step I) If $R=$ completion of an algebra of finite type over $C$ with respect to some m-adic topology, then $R$ is isomorphic with some residue ring of $C\left[X_{1}, \cdots, X_{n}\right]\left[\left[Y_{1}, \cdots, Y_{m}\right]\right]=C[\underline{X}][[\underline{Y}]]$, where the $X_{i}$ 's and the $Y_{j}$ 's are variables ([7], chap. II, (17.5)). So we can assume that $R=C[\underline{X}][[\underline{Y}]]$.

Step II) $R$ is excellent if and only if $R / \mathfrak{\Re}$ is excellent for every prime $\mathfrak{P}$ of height 0 , which lies over some prime $\mathfrak{p}$ of $C$. So we can assume that $C$ is a domain.

Step III) If $\tilde{C}=$ derived normal ring of $C$, then $\tilde{R}=\tilde{C}[\underline{X}][[\underline{Y}]]$ is a finite $R$-module. By [1], theorem 3.1 and cor. 3.2, we can assume that $\tilde{C}=C=$ Dedekind domain.

Step IV) By proposition 4 of n. 2 it is enough to show that $R$ is $J-2$, since $C[\underline{X}]$ is an excellent ring. 
Step V) Assume that $\mathfrak{\beta} \in \operatorname{Spec}(R), \mathfrak{P} \cap C \neq(0)$. Then $R / \mathfrak{P}$ is excellent by [8], theorem 4 . In particular we are done in this case with $J-2$ property.

Step VI) Let us check property $J-2$ when $\mathfrak{B} \in \operatorname{Spec}(R), \mathfrak{P} \cap C=(0)$ and there exists a maximal ideal $\mathfrak{M}$ containing $\mathfrak{B}$ such that $\mathfrak{M} \cap C=(0)$. Since $R / \mathfrak{P}$ has characteristic 0 we have only to show that $\operatorname{Reg}(R / \mathfrak{P})$ contains a non empty open set. Let us observe the following facts:

(i) $R_{\mathfrak{n}} \supseteq L=$ fraction field of $C$;

(ii) $\operatorname{char}(L)=0$;

(iii) $R / \mathfrak{M}$ is algebraic over $L$;

(iv) $\operatorname{dim}\left(R_{\mathfrak{m}}\right)=\mathrm{ht}(\mathfrak{M})=n+m$ (proposition 2, together with the fact that $\mathfrak{M}$ is generated by the $Y_{i}$ 's and a system of generators of $\mathfrak{M}$ modulo $(\underline{Y}))$;

(v) $\operatorname{Der}_{L}\left(R_{\mathfrak{m}}\right)$ contains $n+m$ independent derivations, i.e. the partial ones.

Therefore $\operatorname{rk}\left(\operatorname{Der}_{L}\left(R_{\mathfrak{m}}\right)\right)=n+m=\operatorname{dim}\left(R_{\mathfrak{n}}\right)$, which means that $R_{\mathfrak{n}}$ is a ring of analytic type over $L$ ([3], definition (2.4)). Hence, by [3], theorem (2.5), there are $f_{1}, \cdots, f_{r} \in \mathfrak{P}$ and $D_{1}, \cdots, D_{r} \in \operatorname{Der}_{L}\left(R_{\mathfrak{m}}\right)$ such that:

(i ) $r=\operatorname{ht}(\mathfrak{P})$;

(ii) $\mathfrak{P} R_{\Re}=\sum_{1}^{r} f_{i} R_{\Re}$;

(iii) $d=\operatorname{det}\left(D_{i}\left(f_{j}\right)\right) \notin \mathfrak{P}$.

Moreover the $D_{i}$ 's can be chosen among the partial derivatives, so that they map $R$ into $R$. We can easily conclude that $R_{d} / \mathfrak{\beta} R_{d}$ is a regular domain, i.e. that $\operatorname{Reg}(R / \mathfrak{\Re})$ contains a non empty open set.

Step VII) Let us check property $J-2$ in the following case: $\mathfrak{B} \in \operatorname{Spec}(R), \mathfrak{B} \cap C=(0)$, but, for every maximal ideal $\mathfrak{M}$ containing $\mathfrak{P}$, we have that $\mathfrak{M} \cap C=\mathfrak{p} \neq(0)$. Therefore $\left(\mathfrak{p}, f_{1}(\underline{X}), \cdots, f_{n}(\underline{X}),(\underline{Y})\right.$ ) is a system of generators for $\mathfrak{M}$, if the $f_{i}$ 's are polynomials whose images modulo $\mathfrak{p}$ generate $\mathfrak{M}$ modulo $(\mathfrak{p},(Y))$. Put: $K=C / \mathfrak{p}$. If $K$ is finite, then it is perfect and $\operatorname{Reg}(R / \mathfrak{P})$ contains a non empty open set as it is shown in [5], theorem (2.1) (precisely the argument at the end of the proof of that theorem). So we can assume that $K$ is an infinite field: this means that a necessary condition is fulfilled to allow us to use the concept of "a ring of analytic type over the discrete valuation ring $C_{\mathfrak{p}}$ " in the sense of [5], definition (1.1).

By proposition 3 we can choose $\tilde{K}=$ finite extension of $K$ such that 
$\overline{\mathfrak{M}}=\mathfrak{M}$ modulo $(\mathfrak{p},(\underline{Y}))=\left(\bar{f}_{1}, \cdots, \bar{f}_{n}\right) K[\underline{X}]$ has an algebraic zero $\left(\bar{a}_{1}, \cdots, \bar{a}_{n}\right)$ belonging to $\tilde{K}^{n}$ and $\overline{\mathfrak{M}} \subseteq \tilde{\overline{\mathfrak{M}}}=\left(X_{1}-\bar{a}_{1}, \cdots, X_{n}-\bar{a}_{n}\right) \tilde{K}[X]$.

By proposition $1, \tilde{K}$ can be lifted to a finite module over $C$, say $\tilde{C}$, such that $C \subseteq \tilde{C}, \tilde{C}=$ Dedekind domain, $\tilde{C} / \tilde{\mathfrak{p}}=\tilde{K}$, for some $\tilde{\mathfrak{p}}$ lying over $\mathfrak{p}$.

Put: $\tilde{M}=\left(\tilde{\mathfrak{p}}, X_{1}-a_{1}, \cdots, X_{n}-a_{n},(\underline{Y})\right) \tilde{C}[\underline{X}][[\underline{Y}]]$, where the $a_{i}$ 's are arbitrary inverse images of the $\bar{a}_{i}$ 's modulo $\tilde{\mathfrak{p}}$ (and belong to $\tilde{C}$ ).

Then we have: $\mathfrak{R} \tilde{R} \subseteq \mathfrak{M} \tilde{R} \subseteq \tilde{M}$, where $\tilde{R}=\tilde{C}[X][[\underline{Y}]]$. Therefore we can choose $\widetilde{\mathfrak{D}} \in \operatorname{Spec}(\tilde{R})$ such that: (i) $\mathfrak{B} \tilde{R} \subseteq \tilde{\mathfrak{D}} \subseteq \tilde{\mathfrak{M}}$; (ii) $\widetilde{\mathfrak{D}}$ is minimal over $\mathfrak{R} \tilde{R}$. Then we obtain: 1) $\tilde{\mathfrak{D}} \cap R=\mathfrak{\beta}$ (going down theorem); 2) $\widetilde{\beth} \cap \tilde{C}=(0)$ (by 1)). Since we have the finite inclusion $R / \mathfrak{R} \longrightarrow \tilde{R} / \tilde{\mathfrak{D}}$ we can, by [1], corollary 2.2 , show that $\operatorname{Reg}(\tilde{R} / \tilde{\beth})$ contains a non empty open set, to deduce the same property for $R / \mathfrak{\beta}$.

Let us observe the following facts: (i) $\tilde{R}_{\tilde{\mathbb{M}}}$ is a regular local ring containing $\tilde{C}_{\tilde{p}}$; (ii) $\partial / \partial Y_{i}$ and $\partial / \partial\left(X_{i}-a_{i}\right)=\partial / \partial X_{i}$, for every $i$, maps $\tilde{R}_{\tilde{\mathfrak{M}}}$ into itself.

Therefore $\tilde{R}_{\tilde{\mathfrak{M}}}$ is a ring of analytic type over $\tilde{C}_{\tilde{p}}$, in the sense of [4], definition (2.4). By [4], theorem (1.7), we can choose among the partial derivatives $r$ derivations $D_{1}, \cdots, D_{r}$ (with $r=\operatorname{ht}(\widetilde{\mathfrak{L}})$ ) and $g_{1}$, $\left.\cdots, g_{r} \in \widetilde{\mathfrak{D}}\right)$ such that $d=\operatorname{det}\left(D_{i}\left(g_{j}\right)\right) \notin \widetilde{\mathfrak{D}}$. Finally we see that $\operatorname{Reg}(\tilde{R} / \widetilde{\mathfrak{D}})$ contains the non empty open set $\tilde{R}_{d} / \widetilde{\beth} \tilde{R}_{d}$.

Step VIII) The preceding steps show that $R$ is quasi excellent. Since it is regular it is also universally catenary, hence excellent.

\section{REFERENCES}

[1] Greco, S. Two theorems on excellent rings, Nagoya J. vol. 60, (1976).

[2] Grothendieck, A. E.G.A. Publ. I.H.E.S., Paris, 1960.

[ 3 ] Matsumura, H. Commutative Algebra, Benjamin, New York, 1970.

[ 4 ] Matsumura, H. Formal power series rings over polynomial rings, Number Theory Algebraic Geometry and Commutative Algebra in honour of Y. Akizuki, Tokyo, 1973.

[ 5 ] Nomura, M. Formal power series rings over polynomial rings. II, Number Theory Algebraic Geometry and Commutative Algebra in honour of Y. Akizuki, Tokyo, 1973.

[6] Marot, J. Sur les anneaux universellement japonais, C.R. Acad. Sc. Paris, 1973.

[ 7 ] Nagata, M. Local Rings, Interscience, New York, 1962.

[8] Valabrega, P. On the excellent property for power series rings over polynomial rings, J. Math. of Kyoto Univ., vol. 15, No. 2, (1975).

[9] Zariski and Samuel Commutative Algebra, Van Nostrand, New York, 1960.

Istituto Matematico

Politecnico di Torino 